\title{
An unexpectedly low oscillator strength as the origin of the Fe XVII emission problem
}

\author{
S. Bernitt ${ }^{1}$, G. V. Brown ${ }^{2}$, J. K. Rudolph ${ }^{1,3}$, R. Steinbrügge ${ }^{1}$, A. Graf ${ }^{2}$, M. Leutenegger ${ }^{4,5}$, S. W. Epp ${ }^{1,6}$, S. Eberle ${ }^{1}$, K. Kubiček ${ }^{1} \dagger$, \\ V. Mäckel ${ }^{\dagger} \uparrow$, M. C. Simon ${ }^{7}$, E. Träbert ${ }^{2}$, E. W. Magee ${ }^{2}$, C. Beilmann ${ }^{1}$, N. Hell ${ }^{2,8}$, S. Schippers ${ }^{3}$, A. Müller $^{3}$, S. M. Kahn $^{9}$, \\ A. Surzhykov ${ }^{10,11}$, Z. Harman ${ }^{1,12}$, C. H. Keitel ${ }^{1}$, J. Clementson ${ }^{2}$, F. S. Porter ${ }^{4}$, W. Schlotter ${ }^{13}$, J. J. Turner $^{13}$, J. Ullrich $^{1} \dagger$, \\ P. Beiersdorfer ${ }^{2} \&$ J. R. Crespo López-Urrutia ${ }^{1}$
}

Highly charged iron ( $\mathrm{Fe}^{16+}$, here referred to as $\left.\mathrm{Fe} \mathrm{XVII}\right)$ produces some of the brightest $\mathrm{X}$-ray emission lines from hot astrophysical objects $^{1}$, including galaxy clusters and stellar coronae, and it dominates the emission of the Sun at wavelengths near 15 angströms. The Fe XVII spectrum is, however, poorly fitted by even the best astrophysical models. A particular problem has been that the intensity of the strongest Fe XVII line is generally weaker than predicted $^{2,3}$. This has affected the interpretation of observations by the Chandra and XMM-Newton orbiting X-ray missions ${ }^{1}$, fuelling a continuing controversy over whether this discrepancy is caused by incomplete modelling of the plasma environment in these objects or by shortcomings in the treatment of the underlying atomic physics. Here we report the results of an experiment in which a target of iron ions was induced to fluoresce by subjecting it to femtosecond X-ray pulses from a free-electron laser ${ }^{4}$; our aim was to isolate a key aspect of the quantum mechanical description of the line emission. Surprisingly, we find a relative oscillator strength that is unexpectedly low, differing by $3.6 \sigma$ from the best quantum mechanical calculations. Our measurements suggest that the poor agreement is rooted in the quality of the underlying atomic wavefunctions rather than in insufficient modelling of collisional processes.

Past laboratory measurements using magnetic fusion devices ${ }^{5,6}$ have ruled out some of the astrophysical explanations for the weakness of the strongest Fe XVII line (labelled 3C when first observed in the Sun $40 \mathrm{yr} \mathrm{ago}^{7}$; see Fig. 1), including absorption and scattering by a large

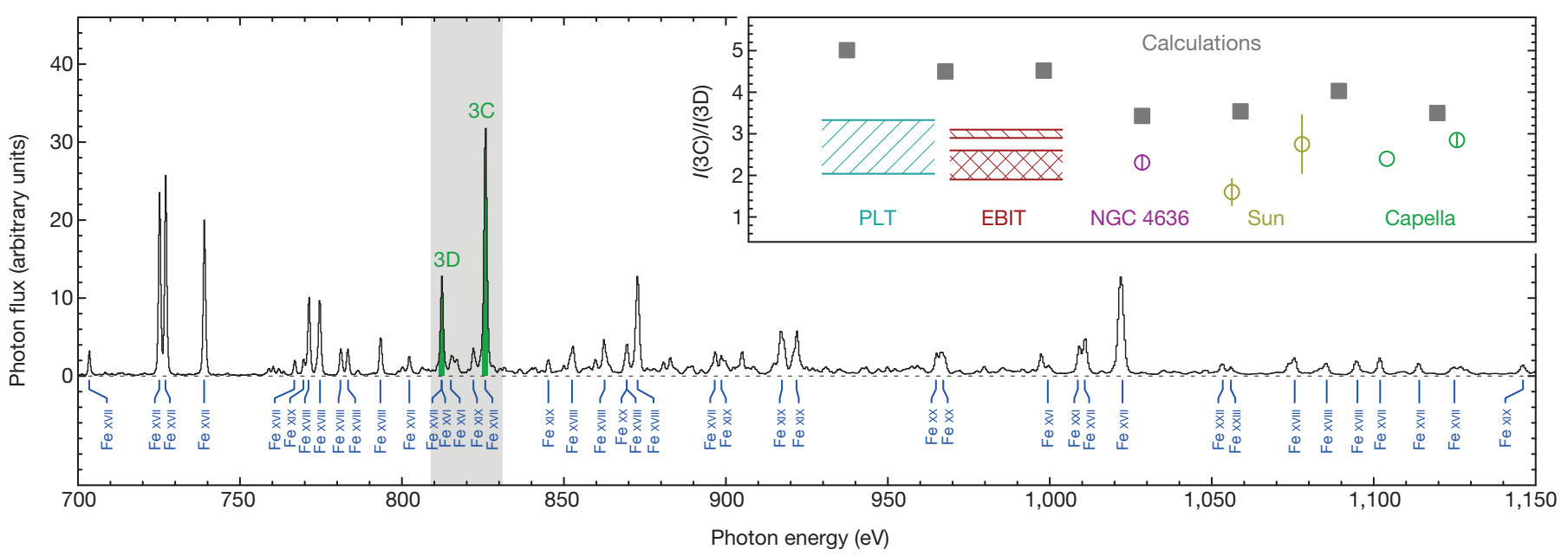

Figure $1 \mid \mathrm{X}$-ray spectrum of Capella, and measured as well as calculated values of the $3 \mathrm{C} / 3 \mathrm{D}$ intensity ratio. Main panel, the spectrum of Capella $(\alpha$ Aurigae) observed by the Chandra X-ray Observatory ${ }^{25}$ is dominated by lines from iron ions of different charge, as indicated by the blue labels. Here, the detected photon flux in arbitrary units is plotted versus the photon energy. Fe Xvir indicates emission from $\mathrm{Fe}^{16+}$ (that is, iron from which 16 electrons were removed), Fe XVIII refers to emission from $\mathrm{Fe}^{17+}$, and so on. The energy range covered by the present measurement is shaded in grey, corresponding to wavelengths near $15 \mathrm{~A}$. The Fe XVII lines $3 \mathrm{C}$ and $3 \mathrm{D}$ are highlighted in green and refer to the $\left(2 p^{5}\right)_{1 / 2}(3 d)_{3 / 2}(J=1) \rightarrow 2 p^{6}(J=0)$ and $\left(2 p^{5}\right)_{3 / 2}(3 d)_{5 / 2}(J=1) \rightarrow 2 p^{6}$ $(J=0)$ transitions at $826 \mathrm{eV}$ and $812 \mathrm{eV}$, respectively. The Fe XVII emission dominates astrophysical spectra for temperatures between about $2 \mathrm{MK}$ and $8 \mathrm{MK}$ (ref. 1). Inset, illustration of some of the available calculated values ${ }^{12,16,21-24}$ and various astrophysical and laboratory observations. Ranges of measured values from the Princeton Large Torus (PLT) tokamak ${ }^{5,6}$ and from the Livermore electron beam ion trap (EBIT) ${ }^{10,26}$ are shown together with astrophysical values from NGC $4635^{3}$, the Sun ${ }^{27,28}$ and Capella ${ }^{2,29}$. Where available, $1 \sigma$ error bars are shown for the astrophysical values. The EBIT range splits into two parts: the upper range (singly hatched) is for measurements where only Fe XVII ions were considered; the lower range (cross hatched) was measured when various amounts of Fe XVI ions were co-mixed with Fe XVII ions. In the latter case, Fe Xvi lines blend with line 3D, increasing its apparent line strength.

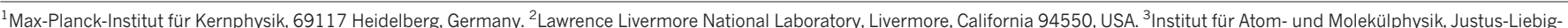

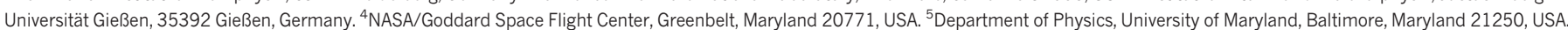

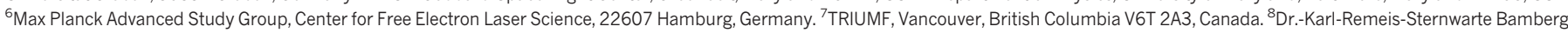

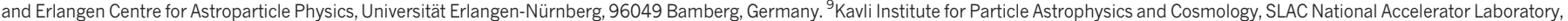

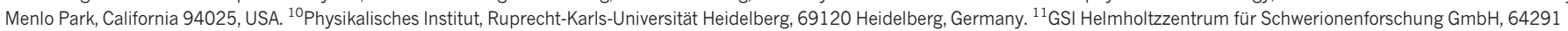

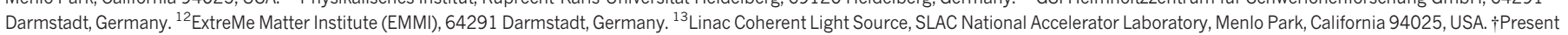
addresses: Max-Planck-Institut für biophysikalische Chemie, 37077 Göttingen, Germany (K.K.); RIKEN, Wako, Saitama 351-0198, Japan (V.M.); Physikalisch-Technische Bundesanstalt, 38116 Braunschweig, Germany (J.U.) 


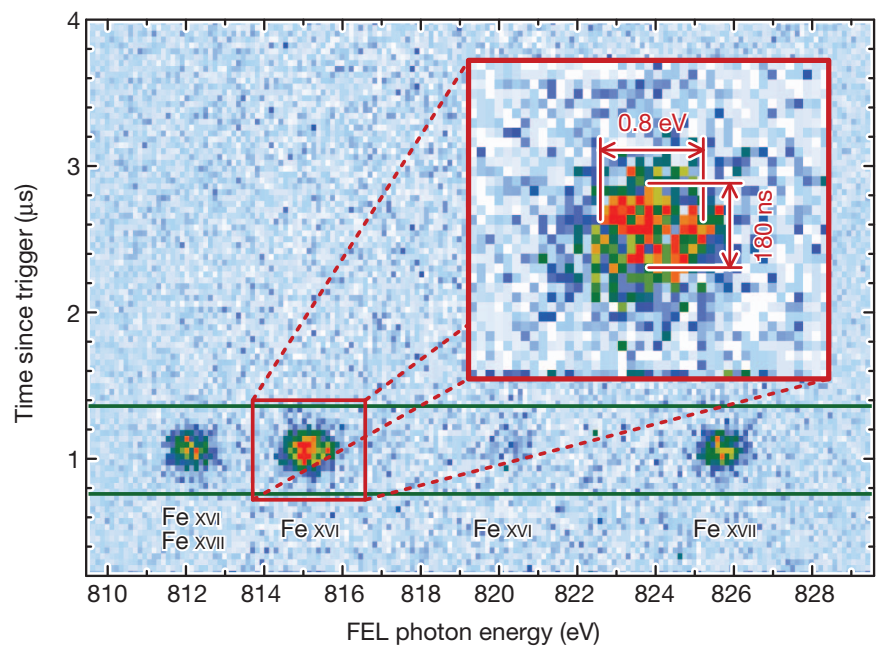

Figure $2 \mid$ Time coincidence between the free-electron laser (FEL) pulse and detected X-ray fluorescence. The horizontal axis shows the FEL photon energy, the vertical axis shows the time after a trigger signal, which is provided approximately $1 \mu$ s before the FEL fires. The colour code indicates the photon count rate per time and energy, from 0 (white) to 15 (red) in $\mathrm{Hz}_{\mu \mathrm{s}}{ }^{-1} \mathrm{eV}^{-1}$. The FEL energy was continuously scanned (200 minutes) from approximately $809 \mathrm{eV}$ to $829 \mathrm{eV}$. Fluorescence is seen from both Fe XVI and Fe XVII, but it is observed only in a small time window following the FEL pulses. The red box (magnified in the inset) shows one of the resonances with the corresponding resolutions in time $(180 \mathrm{~ns})$ and photon energy $(0.8 \mathrm{eV})$. The photon energy resolution is determined by the intrinsic energy resolution of the FEL. Because the radiative decay times of the transitions of interest are below a picosecond, all fluorescence photons resulting from a single, femtosecond LCLS pulse are registered as simultaneous by the high-purity Ge detector used to detect the fluorescence X-rays, and the time window is solely determined by the time resolution (180 ns) of the detector. By gating over a 600-ns-wide time region (indicated by the green lines), we included essentially all fluorescence photons, while suppressing the background (produced by the interaction of the target ions with the electron beam from FLASH-EBIT) by a factor of 14,000 . The much larger number of photons detected outside this time window was used for background determination with very high statistical significance. The background corrected spectra produced following these procedures are shown in Fig. 3.

column of intervening media ${ }^{8,9}$. Targeted measurements, which used an electron beam to produce and probe the ions, have furthermore found line blends that can increase the apparent intensity of some of the FeXVII lines ${ }^{10}$. Very importantly, such measurements have produced absolute collisional excitation cross-sections ${ }^{11}$, which, like the line intensities, have not been reproduced by atomic calculations at the level needed to model astrophysical spectra. Although such laboratory measurements have produced spectra that agree very well with those observed from celestial sources (see Fig. 1 inset), they have not yet pinpointed the fundamental reason why spectral models do not provide the desired agreement with observations.

The consensus among most atomic theorists has been that the remaining problem lies with the calculation of collisional effects, that is, with deficiencies in atomic scattering calculations of the electronimpact excitation cross-section, and special emphasis was therefore placed on correctly including resonances with the help of sophisticated $\mathrm{R}$-matrix methods ${ }^{12}$. Numerous resolutions of the problem have been proposed $^{13,14}$, including the suggestion that kinetic contributions from other levels, which include radiative cascades, collisional depopulation and photon scattering, might need revision depending on the particular plasma conditions ${ }^{15}$. Another possibility ${ }^{16}$ is that the problem may lie in the incorrect or incomplete description of the mixing between wavefunctions of the same parity and total angular momentum (this mixing can also be viewed as an electron-electron correlation issue). The intensity ratio of the Fe XVII lines $3 \mathrm{C}$ and 3D (see Fig. 1) is the key to

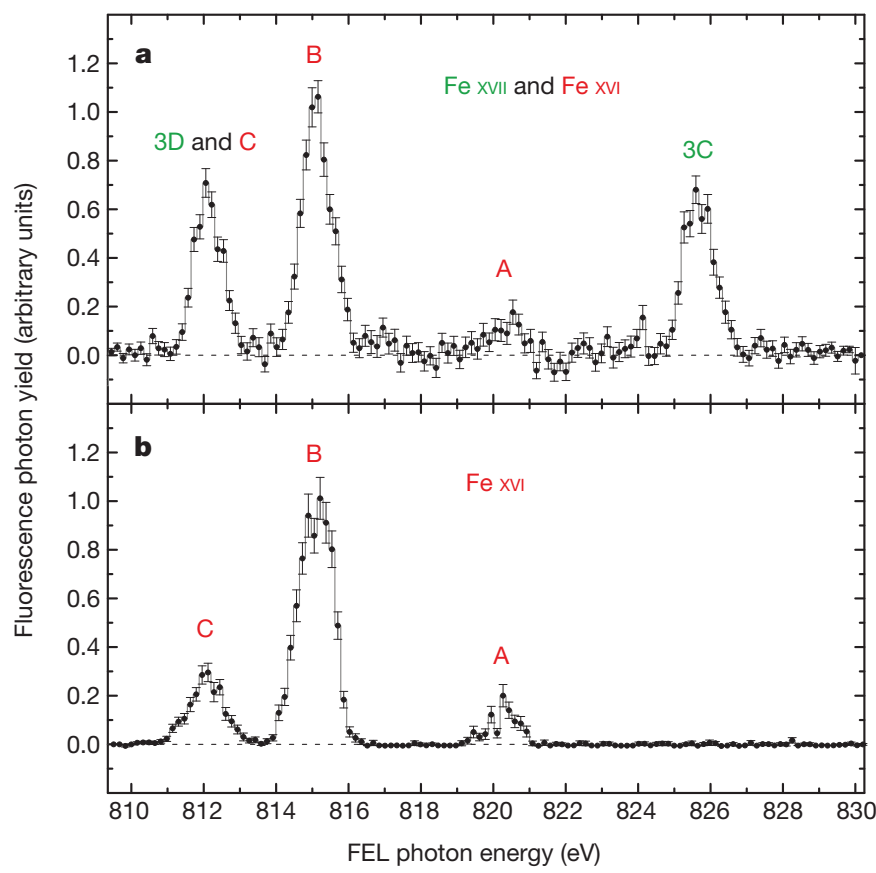

Figure $3 \mid$ Measured X-ray fluorescence spectra. a, A typical fluorescence spectrum of Fe XVI and Fe XVII when both ion species are among the target ions; b, a typical fluorescence spectrum of Fe XVI alone, which is produced in the absence of $\mathrm{Fe}^{16+}$ target ions. Error bars, $1 \sigma$ uncertainties. Fe XVI lines are labelled A, B and C in red; the Fe XVII lines are labelled in green. Both spectra are normalized to the highest peak (line B). The blend of line 3D from Fe XVII and line $\mathrm{C}$ from Fe XVI has to be taken into account when comparing the intensities of $3 \mathrm{C}$ and $3 \mathrm{D}$. The plotted fluorescent photon yield has been adjusted for independently monitored variations in the FEL X-ray intensity as a function of FEL energy.

resolving this controversy. Line 3D is forbidden in the Russell-Saunders or LS-coupling approach to constructing wavefunctions. It only exists because of mixing with $3 \mathrm{C}$ : the more their upper levels mix, the larger the radiative decay rate and thus the larger the oscillator strength of $3 \mathrm{D}$ at the expense of 3C. Because excitation cross-sections vary monotonically with the oscillator strength, as illustrated in Supplementary Information, the increased mixing results in a higher intensity of $3 \mathrm{D}$ relative to that of 3C. Mixing, of course, is not restricted to just these two levels, but occurs among all levels with the same parity and total angular momentum. Because there are an infinite number of atomic levels, theory must introduce approximations to this correlation issue when constructing a particular wavefunction.

In a typical plasma, the intensity ratio of lines 3C and 3D not only reflects the mixing among the electronic levels but also depends on the above-mentioned kinetic contributions and on collisional effects, such as electron-impact excitation, resonance excitation, inner-shell ionization and dielectronic recombination, which need to be integrated over the plasma electron distribution function. Hence, the effect of quantum mechanical mixing cannot be cleanly isolated from the plasma environment, which is described by such quantities as the temperature, density, flows, opacity, spatial gradients and temporal variations, so that the question of why the measured ratio does not agree with theory remains obscure and controversial.

$\mathrm{X}$-ray laser spectroscopy ${ }^{17}$ provides a new experimental method to resolve this controversy because the excitation and fluorescence of the levels depend only on the atomic wavefunctions and not on electronic collisions. In other words, the intensity of the 3C and 3D lines excited by an X-ray laser is directly proportional to the oscillator strength of each line, providing a direct test of the accuracy of the (mixed) wavefunctions that is totally unaffected by the plasma environment, collisional effects and atomic kinetics. For such a measurement we need a 


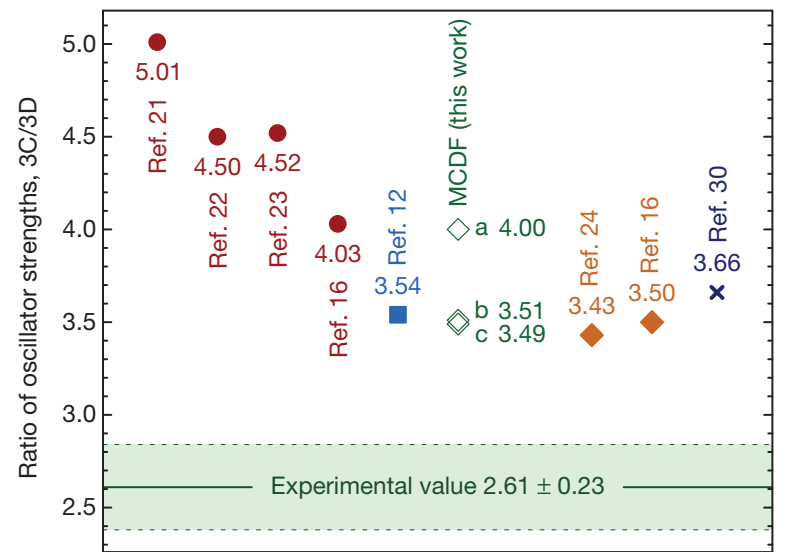

Figure $4 \mid$ Predicted and measured intensity ratios of lines $3 \mathrm{C}$ and 3D. The experimental value and associated $1 \sigma$ uncertainty are shown as a green band. The red circles are values produced by wavefunctions used in typical scattering calculations ${ }^{16,21-23}$. These wavefunctions are not optimized to account for mixing among more than a few electronic levels. The blue square indicates the ratio resulting from wavefunctions employed in a newer scattering calculation that employed more fully converged wavefunctions ${ }^{12}$. The green diamonds illustrate the ratios resulting from wavefunctions generated with the MCDF ansatz (see Supplementary Information) at various levels of completeness (that is, convergence) of mixing among the electronic levels: mixing among nearly 800 levels (a); mixing among nearly 90,000 levels (b); and extrapolation to mixing among 'all' (infinitely many) levels (c). The filled orange diamonds show the ratios arising from wavefunctions produced in calculations using the MBPT ansatz. This ansatz is designed to optimize the treatment of mixing among electronic levels ${ }^{16,24}$ using perturbative methods. Perturbative calculations were carried out to second order only, but the resulting wavefunctions produce ratios of the oscillator strengths that are already lower than those produced by other methods. Higher-order MBPT calculations do not yet exist. The dark blue cross represents the value listed in National Institute for Standards and Technology (NIST) atomic database ${ }^{30}$. The assessed uncertainty (rated 'C+' (or $\pm 18 \%$ ) and 'D' (or $\pm 50 \%$ ) for the relevant Fe XVII lines) associated with the ratio is as large as $50 \%$, which provides overlap with our measurement and enough room for future theoretical improvement using a new quantum mechanical ansatz.

target of $\mathrm{Fe}^{16+}$ and ultrashort, very intense X-ray pulses capable of exciting sufficient numbers of iron ions for detection. The advent of a new kind of ultra-brilliant light source, the free-electron X-ray laser, coupled with a target of trapped, highly charged ions provided by an electron beam ion trap (EBIT) $)^{18}$, now makes such measurements possible.

The experiment was carried out at the Linac Coherent Light Source $(\mathrm{LCLS})^{4}$ free-electron X-ray laser, where the transportable FLASHEBIT $^{19}$ was installed as an end-station in the Soft X-ray Materials Science (SXR) $\operatorname{area}^{20}$ (see Supplementary Information). Efficient detection of the X-ray-induced fluorescence, which took advantage of the femtosecond time structure of the LCLS photon beam, was the key to a successful measurement (Fig. 2). We performed LCLS photon energy scans at EBIT electron beam energies both above and below the threshold for the production of $\mathrm{Fe}^{16+}$ ions. Using this method, we obtained spectra of FeXVI and Fe XVII together, as well as Fe XVI alone (Fig. 3), allowing us to take into account blending of Fe XVI and Fe XVII lines.

The linear polarization of the LCLS photon beam causes anisotropic angular emission patterns for the different X-ray transitions. Therefore, the fluorescence detection efficiency is not the same for all the lines but depends on the detection geometry. Fortunately, lines $3 \mathrm{C}$ and 3D have identical emission patterns, as discussed in Supplementary Information, precluding a possible source of uncertainty. The photon beam intensity monitor of the SXR beamline did not provide absolute photon fluxes. However, we accurately measured the fluorescence intensity ratio of $3 \mathrm{C}$ and $3 \mathrm{D}$ as $2.61 \pm 0.23$, averaged over two independent measurement periods (see Supplementary Information and Supplementary Fig. 2), after accounting for the blend of 3D with the Fe XVI line C.

The measured fluorescence ratio can be directly compared to quantum mechanical calculations of the oscillator strengths of lines $3 \mathrm{C}$ and 3D, as presented in Fig. 4. Computational limitations typically force scattering calculations to rely on some of the poorest approximations for atomic wavefunctions, in which the mixing of the target wavefunctions may be poorly converged. Indeed, typical scattering calculations ${ }^{16,21-23}$ have been based on wavefunctions that predict ratios of the oscillator strengths between 4.0 and 5.0, as summarized in Fig. 4. Some newer R-matrix scattering calculations ${ }^{12}$ have now specifically employed more refined methods to construct wavefunctions, which give a ratio as low as 3.54. Indeed, focused atomic structure calculations designed to produce the best wavefunctions can produce even lower ratios of the oscillator strengths, depending on the quantum mechanical ansatz used. The multiconfiguration Dirac-Fock (MCDF) ansatz (Supplementary Fig. 2) produces a ratio that drops from 4.5, when including mixing among only a reasonable number of wavefunction, to 3.49 when essentially fully converged, that is, when including mixing among a very large number of wavefunctions and thus aiming to approximate the effect of including all levels. The many-body perturbation theory (MBPT) ansatz gives 3.50 or even 3.43 in second order approximation $^{16,24}$ (Fig. 4). The MBPT ansatz might give an even lower ratio, if the calculation were carried out to higher order. However, such a refined MBPT calculation has not yet been done. In other words, our experiment intimates that quantum mechanics has reached a point where the dominant uncertainties lie in the wavefunctions themselves, and a refined MBPT calculation, or yet another different ansatz that includes not only a more complete calculation of correlation effects, but also accounts for quantum electrodynamical terms and the time dependence of atomic dynamics, may be needed to reproduce the experimental value.

Future quantum mechanical calculations may bring the relative oscillator strengths, and thus the ratio of collisional cross-sections associated with them, into closer agreement with our measured value. This would bring the predicted Fe XVII intensity ratios into agreement with those measured in collisional plasmas in the laboratory and observed from X-ray sources in space.

\section{Received 7 May; accepted 27 September 2012}

1. Paerels, F. B. S. \& Kahn, S. M. High-resolution X-ray spectroscopy with Chandra and XMM-Newton. Annu. Rev. Astron. Astrophys. 41, 291-342 (2003).

2. Behar, E., Cottam, J. \& Kahn, S. M. The Chandra iron-L X-ray line spectrum of Capella. Astrophys. J. 548, 966-975 (2001).

3. Xu, H. etal. High-resolution observations of the elliptical galaxy NGC 4636 with the reflection grating spectrometer on board XMM-Newton. Astrophys. J. 579, 600-606 (2002).

4. Emma, P. et al. First lasing and operation of an ångstrom-wavelength free-electron laser. Nature Photon. 4, 641-647 (2010)

5. Beiersdorfer, P. et al. Measurement of the $3 d \rightarrow 2 p$ resonance to intercombination line-intensity ratio in neonlike Fe XVII, Ge XXIII, and Se XXV. Phys. Rev. A 64, 032705 (2001).

6. Beiersdorfer, P. et al. Laboratory measurements of the Fe XVII $2 p-3 s$ and $2 p-3 d$ transitions and comparison with solar and astrophysical observations. Astrophys. J. 610, 616-623 (2004).

7. Parkinson, J. H. New observations of Fe XVII in the solar X-ray spectrum. Astron. Astrophys. 24, 215-218 (1973).

8. Schmelz, J. T., Saba, J. L. R. \& Strong, K. T. Resonance scattering of Fe XVII - a density diagnostic. Astrophys. J. 398, L115-L118 (1992).

9. Waljeski, K. et al. The composition of a coronal active region. Astrophys. J. 429, 909-923 (1994).

10. Brown, G. V. et al. Diagnostic utility of the relative intensity of $3 \mathrm{C}$ to $3 \mathrm{D}$ in Fe XVII. Astrophys. J. 557, L75-L78 (2001)

11. Brown, G. V. etal. Energy-dependent excitation cross section measurements of the diagnostic lines of Fe XVII. Phys. Rev. Lett. 96, 253201 (2006)

12. Chen, G.-X. Converged Dirac R-matrix calculation of electron impact excitation of Fe XVII. Phys. Rev. A 76, 062708 (2007)

13. Nikulin, V. K. \& Trzhaskovskaya, M. B. Comment on "Energy-dependent excitation cross section measurements of the diagnostic lines of Fe XVII". Phys. Rev. Lett. 108, 139301 (2012).

14. Brown, G. V. \& Beiersdorfer, P. Brown and Beiersdorfer reply. Phys. Rev. Lett. 108, 139302 (2012). 
15. Fournier, K. B. \& Hansen, S. B. Resolution of the long-standing overprediction of the resonance to intercombination line-intensity ratio in mid-Z neonlike ions. Phys. Rev. A 71, 012717 (2005).

16. Gu, M. F. New benchmark of X-ray line emission models of Fe XVII. Preprint at http://arXiv.org/abs/0905.0519v1 (2009).

17. Epp, S. W. et al. Soft X-ray laser spectroscopy on trapped highly charged ions at FLASH. Phys. Rev. Lett. 98, 183001 (2007)

18. Marrs, R. E., Beiersdorfer, P. \& Schneider, D. The Electron Beam Ion Trap. Phys. Today 47, 27-34 (1994).

19. Epp, S. W. etal. X-ray laser spectroscopy of highly charged ions at FLASH. J. Phys. At. Mol. Opt. Phys. 43, 194008 (2010).

20. Schlotter, W. F. et al. The soft X-ray instrument for materials studies at the linac coherent light source X-ray free-electron laser. Rev. Sci. Instrum. 83, 043107 (2012).

21. Bhatia, A. K. Atomic data and spectral line intensities for the neon isoelectronic sequence (Si V through Kr XXVII). At. Data Nucl. Data Tables 32, 435-469 (1985).

22. Bhatia, A. K. \& Doschek, G. A. Atomic data and spectral line intensities for Ne-like Fe XVII. At. Data Nucl. Data Tables 52, 1-23 (1992).

23. Cornille, M., Dubau, J. \& Jacquemot, S. Radiative and collisional atomic data for neon-like ions. At. Data Nucl. Data Tables 58, 1-66 (1994).

24. Safronova, U. I. et al. Electric-dipole, electric-quadrupole, magnetic-dipole, and magnetic-quadrupole transitions in the neon isoelectronic sequence. Phys. Rev. A 64, 012507 (2001)

25. Huenemoerder, D. P. et al. TGCat: The Chandra transmission grating data catalog and archive. Astron. J. 141, 129 (2011).

26. Brown, G. V. et al. Laboratory measurement and modeling of the Fe XVII X-ray spectrum. Astrophys. J. 502, 1015-1026 (1998).

27. Blake, R. L. et al. Spectral and photometric measurements of solar X-ray emission below $60 \AA$ A. Astrophys. J. 142, 1-12 (1965).

28. McKenzie, D. L. et al. Solar flare X-ray spectra between 7.8 and 23.0 Ångstroms. Astrophys. J. 241, 409-416 (1980)

29. Mewe, R. et al. CHANDRA-LETGS X-ray observations of Capella. Astron. Astrophys 368, 888-900 (2001)

30. Ralchenko, Y. et al. NIST Atomic Spectra Database (ver. 4.1.0) http://physics. nist.gov/asd (National Institute of Standards and Technology; accessed, 27 March 2012).
Supplementary Information is available in the online version of the paper.

Acknowledgements We thank the staff at MPIK, LLNL and SLAC, especially D. Layne (LLNL) who provided technical support. Portions of this research were carried out on the SXR instrument at the LCLS, a division of SLAC National Accelerator Laboratory and an Office of Science user facility operated by Stanford University for the US Department of Energy. The SXR instrument is funded by a consortium whose membership includes the LCLS, Stanford University through the Stanford Institute for Materials Energy Sciences, Lawrence Berkeley National Laboratory, University of Hamburg through the BMBF priority programme, and the Center for Free Electron Laser Science. The present work was performed in part at LLNL under the auspices of the US Department of Energy and supported in part by the Helmholtz Alliance. P.B. performed part of the work reported here while at the Department of Chemistry and the Chemical Physics Program, University of Puerto Rico. A.S. was supported by the Helmholtz association and Z.H. was supported by EMMI. N.H. acknowledges support from BMBF, and E.T., A.M., J.K.R. and S.S. acknowledge support from the Deutsche Forschungsgemeinschaft

Author Contributions J.R.C.L.-U., P.B. and J.U. conceived the project; G.V.B., A.M. and S.M.K. contributed to the original proposal. S.B., J.K.R., R.S., E.W.M, C.B. and J.R.C.L.-U. prepared the FLASH-EBIT for operation at LCLS. C.B. planned the integration of the

FLASH-EBIT at the SXR beamline. S.B., G.V.B., J.K.R., R.S., C.B., A.G., N.H., M.L., S.E., S.W.E., K.K., V.M., M.C.S., S.S., E.T. and J.R.C.L.-U. operated the FLASH-EBIT and detectors. F.S.P. provided technical assistance for the operation of one of the detectors. W.S. and J.J.T. prepared and operated the SXR instruments. A.S., J.C., P.B, Z.H. and C.H.K. performed supporting calculations. R.S. converted raw data for further processing and analysis. S.B. performed the data analysis. S.B., G.V.B., A.G., M.L., S.W.E., S.S., E.T., J.U., P.B. and J.R.C.L.-U. interpreted the results. P.B. wrote the manuscript with input from S.B. and J.R.C.L.-U.; Z.H., C.H.K., S.B., A.S. and P.B. wrote the Supplementary Information. All authors were involved in the discussion of results and commented on the manuscript.

Author Information Reprints and permissions information is available at www.nature.com/reprints. The authors declare no competing financial interests. Readers are welcome to comment on the online version of the paper. Correspondence and requests for materials should be addressed to S.B. (sven.bernitt@mpi-hd.mpg.de). 